\title{
Two computable sets of multipartite entanglement measures
}

\author{
Beatrix C. Hiesmayr, Marcus Huber, and Philipp Krammer \\ Faculty of Physics, University of Vienna, \\ Boltzmanngasse 5, 1090 Vienna, Austria
}

\begin{abstract}
We present two sets of computable entanglement measures for multipartite systems where each subsystem can have different degrees of freedom (so-called qudits). One set, called "separability" measure, reveals which of the subsystems are separable/entangled. For that we have to extend the concept of $k$-separability for multipartite systems to a novel unambiguous separability concept which we call $\gamma_{k}$-separability. The second set of entanglement measures reveals the "kind" of entanglement, i.e. if it is bipartite, tripartite, $\ldots, n$-partite entangled and is denoted as the "physical" measure. We show how lower bounds on both sets of measures can be obtained by the observation that any entropy may be rewritten via operational expressions known as $m$-concurrences. Moreover, for different classes of bipartite or multipartite qudit systems we compute the bounds explicitly and discover that they are often tight or equivalent to positive partial transposition (PPT).
\end{abstract}

Keywords: entanglement measure, multipartite qudit system, separability PACS: 03.67.Mn

\section{INTRODUCTION}

Quantum entanglement is a fascinating property of quantum states that has many important consequences for modern physics. It exhibits aspects that are counter-intuitive to classical physics, like the incompatibility with local realistic theories [1, 2]. For example it turned out that a symmetry violation in particle physics, the $C P$ violation in mixing ( $C$. . charge conjugation, $P \ldots$ parity), is incompatible with any local realistic theory [3, 4] .

Furthermore entanglement is a highly useful resource for quantum information tasks. Thus it makes quantum information theory a conceptually different theory than classical information theory (for an overview see, e.g., Refs. [5, 6, 7]). The characterization of entanglement is, however, a nontrivial mathematical task and not at all completed (for an overview see, e.g., Refs. [8, 9] ). The first concepts were derived for bipartite systems, which are the simplest systems that can contain entanglement. Here many important results were obtained, like the detection and quantification of entanglement for (pure and mixed) bipartite qubits, which can be conclusively performed for any states of such systems. In a finite dimensional Hilbert space the most general quantum states one can think of are multipartite arbitrary dimensional states, i.e. states that describe systems of $n$ subsystems, where each subsystem is ascribed a finite dimensional Hilbert space. Already the classification of entanglement according to possible reversible quantum operations is a nontrivial task, see e.g. Refs. [10, 11, 12] in this context.

There are different approaches to the quantification of multipartite entanglement. A common method is to describe the same state with different entanglement measures, e.g. in terms of bipartite cuts [13] or with different entanglement measures according to invariance classes 
under statistical local operations and classical communication (SLOCC) 12]. Another way is to determine a global entanglement measure for the whole state [14, 15, 16, 17, 18, 19, 20, 21]. Our approach picks up a conception of entanglement that on the one hand differentiates between possible entanglement between any parties sharing the state and on the other hand sums up to a total global entanglement. In this way we can both quantify the entanglement that any parties share with each other, and the "whole" entanglement present in the state.

This would also provide advantages for the description of quantum communication protocols with multipartite entangled states (see, e.g., Refs. [22, 23, 24, 25]). Of course this simple concept already calls for more than one entanglement measure - for different tasks different entanglement measures seem to be appropriate. We want to present two of such possibilities that in our opinion seem to be good candidates, i.e. one revealing the separability property, "the separability measure", and the other one revealing different kinds of entanglement, "the physical measure". Further approaches to quantify multipartite entanglement can be found, e.g., in Refs. 14, 21, 26] and higher dimensional generalizations of bipartite entanglement measures in [27, 28, 29, 30].

The paper is organized as follows: In the first section we define separability of multipartite systems and list the requirements for bipartite entanglement measures. In Sec. III we discuss entropies and introduce the $m$-concurrence which enables computation of bounds on entanglement of mixed states. The next Sec. IV introduces two measures, one for the partial separability and one for what kind of entanglement is present. Then follows a section with further instructive examples to which we applied the two measures. In the appendix we give all proofs of the requirements for these two measures.

\section{BASIC DEFINITIONS}

\section{A. A definition of partial separability and the $\gamma_{k}$-separability}

In multipartite systems the notion of separability can be extended in order to answer the question which particles are joint inseparably. Throughout the paper we assume that partial traces of the multipartite quantum system are only taken over physical subsystems, i.e. over one or more particles. It means that possible information which may result by tracing over certain degrees of freedom of a certain particle/qudit is not taken into account.

A pure multipartite state $|\psi\rangle$ is called $k$-separable if it can be written as [9]

$$
|\psi\rangle=\left|\phi_{1}\right\rangle \otimes\left|\phi_{2}\right\rangle \otimes \cdots \otimes\left|\phi_{k}\right\rangle, \quad k \leq n,
$$

where $n$ is the total number of particles. It is called fully separable iff $k=n$, this is the natural generalization of the separability of bipartite systems. We call a state 1-separable or fully entangled iff $k=1$. This notation of full separability or entanglement can be generalized to mixed states in a straight forward way. If a pure state is not fully separable or fully entangled, it is called partially separable or $k$-separable.

The definition of partial separability for mixed states is more involved. One obvious possibility is the following: A mixed state is called $k$-separable if there exists a decomposition that satisfies [9]

$$
\sigma_{k-s e p}=\sum_{i} p_{i} \rho_{i}^{1} \otimes \rho_{i}^{2} \otimes \cdots \otimes \rho_{i}^{k}, \quad \text { with } \quad p_{i} \geq 0, \sum_{i} p_{i}=1,
$$


where the $\rho_{i}^{j}$ S are states of some number of subsystems and can always be chosen to be pure. The terms in Eq. (2) all have the same $k$, but it is in general not fixed which subsystems are contained in the states $\rho_{i}^{j}$.

For the argumentation in this paper we are interested to fix the subsystems involved in the states $\rho_{i}^{j}$ and therefore extend the $k$-separability definition to the so-called $\gamma_{k}$-separability. For this we introduce the following notation:

$$
\gamma_{k}:=\left\{\left\{\beta_{1}\right\}\left|\left\{\beta_{2}\right\}\right| \cdots \mid\left\{\beta_{k}\right\}\right\} .
$$

Here the sets $\left\{\beta_{j}\right\}$ represent subsystems, i.e. particles, which are inseparably joined.

Instructive example: $|\psi\rangle=|0\rangle_{1} \otimes|0\rangle_{2} \otimes\left|\phi^{+}\right\rangle_{34}$ with $\left|\phi^{+}\right\rangle=\frac{1}{\sqrt{2}}\{|0\rangle \otimes$ $|0\rangle+|1\rangle \otimes|1\rangle\}$. Here the number of particles is $n=4$ and the separability is a 3 -separability with the substructure $\gamma_{3}=\{1|2| 34\}$.

This state is obviously equivalent to $\frac{1}{\sqrt{2}}\{|0000\rangle+|1010\rangle\}$ with the substructure $\gamma_{3}=\{2|4| 13\}$, here just the role of the first and second subsystems are interchanged. Therefore, it is convenient to reorder the subsystems of the state if necessary.

Note that there is a difference between fully entangled and being maximally entangled, which we define as a pure state where all subsystems representing particles are in the maximally mixed state. For example the state $\left|\phi^{+}\right\rangle \otimes\left|\phi^{+}\right\rangle$is not fully entangled, but according to the above definition maximally entangled.

The extension of the $\gamma_{k}$ separability to mixed states is not straightforward as an ambiguity can happen as we explain later in an example.

Definition of $\gamma_{k}$-separability:

To every $\rho$ we associate a separability property, the set $\gamma_{k}$, which is made up of $\left\{\beta_{j}\right\}$, i.e. sets of numbers representing subsystems. A state $\rho$ is called $\gamma_{k}$-separable iff there exists an unambiguous decomposition with maximal $k$ into:

$$
\sigma_{\gamma_{k}-\text { sep }}=\sum_{i} p_{i} \rho_{i}^{\left\{\beta_{1}\right\}} \otimes \rho_{i}^{\left\{\beta_{2}\right\}} \otimes \cdots \otimes \rho_{i}^{\left\{\beta_{k}\right\}}, \quad \text { with } \quad p_{i} \geq 0, \sum_{i} p_{i}=1 .
$$

The following instructive example shows the difference of the $k$-separability and the $\gamma_{k^{-}}$ separability.

Consider the generalized Smolin state [31, 32, 33]

$$
\rho_{\text {Smolin }}=\frac{1}{2^{n}}\left(\mathbb{1}+\sum c_{i} \sigma_{i}^{\otimes n}\right),
$$

where $n$ is an even number, $\sigma_{i}$ are the Pauli matrices and $c_{i}$ are real numbers (see also the instructive example in Sect. IVA). This state can be decomposed into bipartite pure states, i.e. the Bell states. For $n=4$ this would correspond to $\gamma_{k}=\{12,34\}$. This however is not the proper $\gamma_{k}$ separability as any other bipartite cut is also valid, i.e. $\gamma_{k}=\{13,24\}, \gamma_{k}=\{14,23\}$. So the only unambigous set of subsystems is: $\gamma_{k}=\{1234\}$. So in the notion of $\gamma_{k}$-separability, the generalized Smolin states are always completely inseparable ( $\gamma_{1}$-separable), whereas in the notion of $k$-separability they are $\frac{n}{2}$-separable. 


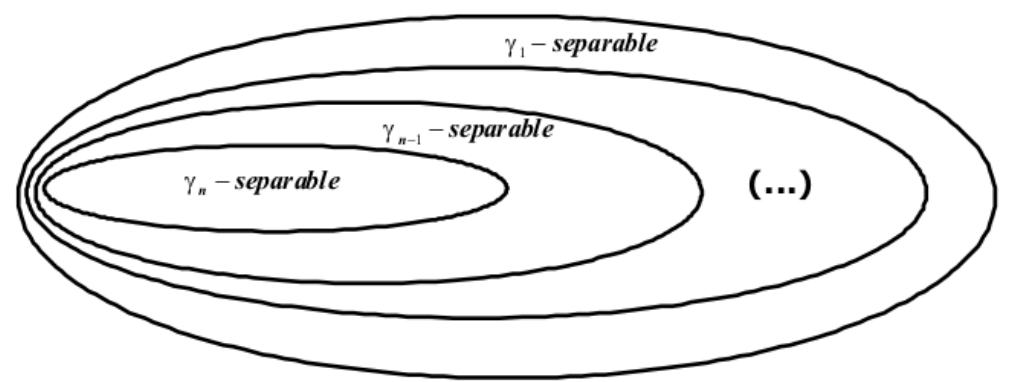

FIG. 1: Here the convexity of $\gamma_{k}$-separability is visualized, i.e. any convex mixture of two $\gamma_{k^{-}}$ separable states, e.g. $\gamma_{k_{1}}$ and $\gamma_{k_{2}}$, is either $\gamma_{k_{1}}-$ or $\gamma_{k_{2}}-$ or $\gamma_{k}$-separable with $k_{1}, k_{2}<k$.

Both views are in a way justified: The $\gamma_{k}$-separability reflects the fact that any further reduction (partial trace) of the state yields a fully separable state (which is independent of notion) and therefore the useful entanglement properties can only be extracted if one uses all contained subsystems. Whereas the $k$-separability reflects how many parties need to join together in order to prepare the state using LOCC. Note again that for pure states the $k$ in $k$-separability and the $k$ in $\gamma_{k}$ are identical.

To sum up, $\gamma_{k}$-separability for pure states is an extension of $k$-separability, it captures which subsystems are involved, and for mixed states it captures an essential novel feature (cf. the above example of the Smolin states) that would be missed by only considering $k$-separability. Another important feature of the $\gamma_{k}$-separability is the convexity in the sense that the mixture of two $\gamma_{k}$-separable states, e.g. $\gamma_{k_{1}}$ and $\gamma_{k_{2}}$, is either $\gamma_{k_{1}}-$ or $\gamma_{k_{2}}-$ or $\gamma_{k}$-separable with $k_{1}, k_{2}<k$. This is visualized in Fig. 1 .

One aim of this paper is to quantify entanglement and classify the $\gamma_{k}$-separability of a given state which is done in Sect. IVA.

\section{B. Proper properties for being entangled}

Now we investigate the question what properties a proper entanglement measure should have. Let us first summarize the conditions which are required for bipartite entanglement measures $E(\rho)$ (Sep is the set of all separable states) [34, 35, 36, 37]:

B1: $E(\rho)>0 \quad \forall \quad \rho \notin S e p$

B2: $E(\rho)=0 \quad \forall \quad \rho \in S e p$

B3: $E\left(\rho^{\otimes n}\right)=n E(\rho)$ (Additivity)

B4: $E\left(\lambda \rho_{1}+(1-\lambda) \rho_{2}\right) \leq \lambda E\left(\rho_{1}\right)+(1-\lambda) E\left(\rho_{2}\right)$ (Convexity)

B5: $E\left(U^{A} \otimes U^{B} \rho\left(U^{A} \otimes U^{B}\right)^{\dagger}\right)=E(\rho)$ (Invariance under local unitary operations)

B6: $\sum_{i} \operatorname{Tr}\left(V_{i} \rho V_{i}^{\dagger}\right) E\left(\frac{V_{i} \rho V_{i}^{\dagger}}{\operatorname{Tr}\left(V_{i} \rho V_{i}^{\dagger}\right)}\right) \leq E(\rho)$ (Non-increasing on average under LOCC), where $V_{i}$ is a separable operator, i.e. of the local form $V_{i}:=A_{i} \otimes B_{i}$. 
For multipartite systems we claim that there cannot be only a single entanglement measure, since it could not correctly quantify the substructure of the $k$-separability or the $\gamma_{k}$-separability and simultaneously reveal which parts of the system are entangled in which way with other parts.

Therefore we propose for multipartite systems a set of entanglement measures $E_{\left\{\alpha_{j}\right\}}$ where the set $\left\{\alpha_{j}\right\}$ denotes subsystems of the whole. As any bipartite system can be seen as a substructure of a bigger system, clearly the same requirements as for bipartite systems applies also to bipartite $E_{\left\{\alpha_{j}\right\}}$. The same should hold true for any tripartite, four-partite, $\ldots$ and so on, entanglement. The sum over the whole set should constitute the total entanglement

$$
E_{t o t}(\rho)=\sum_{j=2}^{n} \sum_{\left\{\alpha_{j}\right\}} E_{\left\{\alpha_{j}\right\}}(\rho) .
$$

It is well known that the entanglement of a pure state can easily be quantified by the entropy of its subsystems [35]. Possible entropy measures are, e.g., the quantum version of Renyi's $\alpha-$ entropies [38]:

$$
S_{\alpha}^{q}:=\frac{1}{1-\alpha} \log _{q} \operatorname{Tr}\left(\rho^{\alpha}\right)
$$

which for $\alpha \rightarrow 1$ equals the famous von Neumann entropy. The logarithmic entropies have the advantage that they imply additivity, for the general cases of probability distributions, for which they were originally intended, as well as for entanglement measures constructed out if it.

Another possibility are the linear entropies

$$
S_{r}(\rho):=\frac{d^{r-1}}{d^{r-1}-1}\left(1-\operatorname{Tr}\left(\rho^{r}\right)\right),
$$

where $d$ is the dimension of $\rho$.

For any multipartite pure state $\rho=|\psi\rangle\langle\psi|$ one can quantify the total entanglement by

$$
E_{t o t}(\rho):=\sum_{s=1}^{n} S\left(\rho_{s}\right)
$$

where $\rho_{s}:=\operatorname{Tr}_{\neg s} \rho$ denotes the reduced density matrix of the respective subsystem $s$ and $S$ is any entropy function. A standard method to generalized this measure for mixed states $\rho=\sum_{i} p_{i}\left|\psi_{i}\right\rangle\left\langle\psi_{i}\right|$ is by constructing the convex roof [39]

$$
E_{t o t}(\rho):=\inf _{\left\{p_{i}, \psi_{i}\right\}} \sum_{i} p_{i} \sum_{s=1}^{n} S\left(\rho_{s}^{i}\right) .
$$

The $\psi_{i}$ are throughout the paper considered as normalized. In general it is not known how to find the infimum, we will show in the next section how with a simple algebraic trick operators can be constructed which allow to compute bounds on the entanglement which turn out to be tight in many cases. 


\section{ENTROPY AND $m$-CONCURRENCE}

The $m$-concurrence was introduced in Ref. [40]. It can be efficiently used to compute bounds for the convex roof extension of the entanglement measures for mixed states. For pure multipartite states it is a simple algebraic rewriting of the entropies of the subsystems in terms of such operators. For the generally mixed subsystems states one can via these operators obtain bounds on the entanglement.

The definition we present here will be slightly modified to the above cited works in oder to yield a simpler and more compact notation. The linear entropy $S_{r=2}$, Eq. (8), of any subsystem $s$ can be rewritten as a sum of terms named concurrences in analogy to Hill and Wootters concurrence [41, 42] and concurrences defined for bipartite systems of arbitrary dimension [28, 29]:

$$
\begin{aligned}
S_{2}\left(\rho_{s}\right) & =\frac{d}{d-1}\left(1-\operatorname{Tr}\left(\rho_{s}^{2}\right)\right) \\
& =\sum_{\alpha} C_{\mathbf{s} \alpha}^{2}+\sum_{\alpha} \sum_{\beta} C_{\mathbf{s} \alpha \beta}^{2}+(\ldots)+\sum_{\alpha} \sum_{\beta} \cdots \sum_{\omega} C_{\mathbf{s} \alpha \beta \cdots \omega}^{2} .
\end{aligned}
$$

This $m$-concurrences $C_{\left\{\alpha_{m}\right\}}^{2}$ containing $m$-indices are obtained using $m$-flip operators in the following way:

$$
C_{\mathbf{s} \alpha \beta \cdots \omega}^{2}:=\sum_{O_{C}}\left|\left\langle\psi|\underbrace{\left(A\left|\left\{i_{n}\right\}\right\rangle\left\langle\left\{i_{n}\right\}|\mathbb{1}-B|\left\{i_{n}\right\}\right\rangle\left\langle\left\{i_{n}\right\}\right| A B\right)}_{O_{C}}| \psi^{*}\right\rangle\right|^{2}
$$

where

$$
\begin{aligned}
& A:=\left(\sigma_{k_{K} l_{K}}^{K \in\{\mathbf{s} \alpha \cdots \omega\}}, \mathbb{1}^{K \notin\{\mathbf{s} \alpha \beta \cdots \omega\}}\right) \\
& B:=\left(\sigma_{k_{K} l_{K}}^{K=\mathbf{s}}, \mathbb{1}^{K \neq \mathbf{s}}\right)
\end{aligned}
$$

and

$$
\sum_{O_{C}}:=\sum_{k_{K}=0}^{d_{K}-1} \sum_{l_{K}>k_{K}} \sum_{\left\{i_{n}\right\}}
$$

Here $K$ denotes the respective subsystem and the flip operators are defined for a qudit system of dimension $d$ in the following way:

$$
\sigma_{k l}^{d \times d}|k\rangle=|l\rangle, \quad \sigma_{k l}^{d \times d}|l\rangle=|k\rangle \quad \text { and } \quad \sigma_{k l}^{d \times d}|t\rangle=0 \quad \forall t \neq k, l .
$$

Note that these are the symmetric generalized Gell-Mann operators (see, e.g., Refs. [43, 44]; generalized Gell-Mann operators are the SU(N) generators). In order to obtain Renyi's entropy we use the relationship between this entropy (7) and the linear entropy (8)

$$
S_{\alpha}^{q}(\rho)=\frac{1}{1-\alpha} \log _{q}\left(\operatorname{Tr}\left(\rho^{\alpha}\right)\right)=\frac{1}{1-\alpha} \log _{q}\left(1-\frac{d^{\alpha-1}-1}{d^{\alpha-1}} S_{\alpha}(\rho)\right) .
$$

Note that one can also obtain the von Neumann entropy by means of the $m$-concurrence. Obviously, that requires computation of all $S_{k}$ from $\alpha=2$ to $\alpha=d$. We choose Renyi's entropy with $\alpha=2$ and $q=2$. In the following we write $S$ for $S_{2}^{2}$. 
As shown in [31, 40], the advantage of rewriting the entropies by means of operators is that it is known how to derive bounds. We present here a way analogous to the method introduced for the concurrence for bipartite systems in Ref. [30]. For that we define a flipped density matrix

$$
\widetilde{\rho}_{O_{C}}:=\left(O_{C}+O_{C}^{\dagger}\right) \rho^{*}\left(O_{C}+O_{C}^{\dagger}\right),
$$

where the conjugation is taken in the computational basis. By calculating the square root of the eigenvalues of $\rho \widetilde{\rho}_{O_{C}}$, which we denote as $\lambda_{i}^{O_{C}}$, the bounds for the concurrence are given by:

$$
C_{\mathbf{s} \alpha \beta \cdots \omega}(\rho) \geq \max \left\{0, \sum_{O_{C}}\left(2 \max _{\lambda_{i}^{O_{C}}}\left(\left\{\lambda_{i}^{O_{C}}\right\}\right)-\sum_{i} \lambda_{i}^{O_{C}}\right)\right\} .
$$

\section{MULTIPARTITE ENTANGLEMENT MEASURES}

In this section we propose the two sets of multipartite entanglement measures. First, we introduce the separability measure that is based on the $\gamma_{k}$-separability, and second, the physical measure that reveals the "kind" of entanglement between subsystems (bipartite, tripartite,... entanglement).

\section{A. Separability measure}

In the following we assume that the total state $\rho$ is pure. For the generalized multipartite set of entanglement measures there are a few alternatives, we propose the following generalization:

S1a: $E_{\text {tot }}(\rho)=\sum_{s=1}^{n} S\left(\rho_{s}\right):=\sum_{\left\{\alpha_{j}\right\}} E_{\left\{\alpha_{j}\right\}}>0 \quad \forall \rho$ with $k<n$

S1b: $E_{\text {tot }}(\rho)=0 \quad \forall \rho \quad$ with $k=n$

S2: $E_{\left\{\alpha_{j}\right\}}(\rho)>0 \quad \forall\left\{\alpha_{j}\right\} \in \gamma_{k}$ and $\left|\left\{\alpha_{j}\right\}\right| \geq 2$

S3: $E_{\left\{\alpha_{j}\right\}}(\rho)=0 \quad \forall\left\{\alpha_{j}\right\} \notin \gamma_{k} \quad$ or $\quad\left|\left\{\alpha_{j}\right\}\right|=1$

S4: $E_{\left\{\alpha_{j}\right\}}\left(\rho^{\otimes n}\right)=n E_{\left\{\alpha_{j}\right\}}(\rho)$ (additivity on copies of the same state)

S5: $E_{\left\{\alpha_{j}\right\}}\left(U_{\alpha_{1}} \otimes U_{\alpha_{2}} \otimes \cdots \otimes U_{\alpha_{j}} \rho\left(U_{\alpha_{1}} \otimes U_{\alpha_{2}} \otimes \cdots \otimes U_{\alpha_{j}}\right)^{\dagger}\right)=E_{\left\{\alpha_{j}\right\}}(\rho)$ (invariance under local unitary operations)

S6: $E_{t o t}\left(\rho_{1} \otimes \rho_{2}\right)=E_{t o t}\left(\rho_{1}\right)+E_{t o t}\left(\rho_{2}\right)$ (additivity on tensor products of arbitrary states) With a measure that fulfills all this requirements one obtains the $\gamma_{k}$-separability and, moreover, the quantified information content of a given state.

According to our notation of $\gamma_{k}$-separability a pure state of three qubits can be entangled in four different ways, $\{1 \mid 23\} ;\{12 \mid 3\} ;\{13 \mid 2\} ;\{123\}$, hence we have four different entanglement measures, which we define in an intuitive way by

$$
\begin{aligned}
E_{12}: & =\left\{S\left(\rho_{1}\right)+S\left(\rho_{2}\right)\right\} \cdot \delta\left[S\left(\rho_{12}\right), 0\right] \\
E_{13}: & =\left\{S\left(\rho_{1}\right)+S\left(\rho_{3}\right)\right\} \cdot \delta\left[S\left(\rho_{13}\right), 0\right] \\
E_{23}: & =\left\{S\left(\rho_{2}\right)+S\left(\rho_{3}\right)\right\} \cdot \delta\left[S\left(\rho_{23}\right), 0\right] \\
E_{123}: & =S\left(\rho_{1}\right)+S\left(\rho_{2}\right)+S\left(\rho_{3}\right)-E_{12}-E_{13}-E_{23}
\end{aligned}
$$


with

$$
\begin{aligned}
& \delta\left[S\left(\rho_{\left\{\alpha_{j}\right\}}\right), 0\right]=1 \quad \text { if } \quad S\left(\rho_{\left\{\alpha_{j}\right\}}\right)=0 \\
& \delta\left[S\left(\rho_{\left\{\alpha_{j}\right\}}\right), 0\right]=0 \text { if } S\left(\rho_{\left\{\alpha_{j}\right\}}\right)>0 .
\end{aligned}
$$

Instructive example for three qubits: For the state

$$
|\tilde{\phi}\rangle=\frac{1}{N}\left\{p|G H Z\rangle+(1-p)\left|\phi^{+}\right\rangle \otimes\{\cos \alpha|0\rangle+\sin \alpha|1\rangle\}\right\}
$$

with $|G H Z\rangle=\frac{1}{\sqrt{2}}\{|000\rangle+|111\rangle\}$ we expect for $p=0$ that it is 2separable with $\gamma_{2}=\{12 \mid 3\}$ (in detail $E_{12}=2, E_{13}=E_{23}=E_{123}=0$ ) and for $p=1$ it is 1 -separable (=fully entangled) $\left(E_{123}=1, E_{12}=\right.$ $\left.E_{13}=E_{23}=0\right)$. For values of $p \in\{0,1\}$ it depends on $\alpha$. With the separability measure this can be easily calculated:

(a) If we want $E_{12}$ to vanish, then $S\left(\rho_{12}\right)$ has to be zero, this can be obtained for $p \leq 0.58$ and $\cos ( \pm \alpha)=\frac{p^{2}-p \pm \sqrt{p^{4}-6 p^{3}+11 p^{2}-8 p+2}}{2\left(p^{2}-2 p+1\right)}$. The state is fully separable, except for $p=0.5$ where $S\left(\rho_{12}\right)=0$, but also $S\left(\rho_{1}\right), S\left(\rho_{2}\right), S\left(\rho_{3}\right)=0$.

(b) No $\alpha$ and $p$ exist such that $S\left(\rho_{13}\right)$ or $S\left(\rho_{23}\right)$ vanish, thus $E_{13}=$ $E_{23}=0$.

(c) As $E_{13}$ and $E_{23}$ are always zero, the state is either $\gamma_{1}=\{123\}$ separable $\left(E_{123}>0\right)$ or $\gamma_{2}=\{12 \mid 3\}$-separable $\left(E_{12}>0\right)$; except for $p=0.5$ and the above $\alpha$ then the state is fully separable $\gamma_{3}=\{1|2| 3\}$.

(d) If the GHZ state is interchanged with a $\mathrm{W}$ state the state is for all $p$ and $\alpha 3$-separable except for $p=0$, where it is clearly $\gamma_{2}{ }^{-}$ separable.

The separability measure can be generalized for multipartite qudit systems as

$$
E_{\left\{\alpha_{j}\right\}}:=\sum_{s \in\left\{\alpha_{j}\right\}}\left(S\left(\rho_{s}\right)-\sum_{\left\{\beta_{j}\right\} \subset\left\{\alpha_{j}\right\}} E_{\left\{\beta_{j}\right\}}\right) \cdot \delta\left[S\left(\rho_{\left\{\alpha_{j}\right\}}\right), 0\right],
$$

where

$$
\sum_{\left\{\alpha_{j}\right\}} E_{\left\{\alpha_{j}\right\}}=E_{t o t}(\rho)
$$

This is an important feature as any violation of this necessary requirement would imply either neglecting or over-quantifying of the information content. As is proven in the Appendix VIIIA this proposed set of measures meets all requirements S1-S6.

The separability measure provides a set of entanglement measures for pure states. In principle it can be extended to mixed states using the convex roof method,

$$
E_{\left\{\alpha_{j}\right\}}(\rho):=\inf \sum_{i} p_{i} E_{\left\{\alpha_{j}\right\}}\left(\left|\psi_{i}\right\rangle\left\langle\psi_{i}\right|\right)
$$


Since there still is no method to calculate the convex roof for arbitrary states, the proposed measure is computable only for pure states or mixed states for which the bound of the $m^{-}$ concurrences are exact or for states where we can know if all involved entropies vanish or not. Clearly, if one cannot execute the defined Kronecker $\delta$ 's exactly, the computation of the measure may fail. Moreover, when applying the separability measure for mixed states, one does in general not obtain e.g. whether the state at all is entangled as the the following example shows:

Instructive example for even number of qubits: Consider the generalized Smolin state $\rho_{\text {Smolin }}=\frac{1}{2^{n}}\left(\mathbb{1}+\sum c_{i} \sigma_{i}^{\otimes n}\right)$, where $n$ is a even number. This state is mixed (except for $n=2$ and $|\vec{c}|=3$ ) and the states of all subsystems are maximally mixed. Therefore all $E_{12 \ldots j}$ with $j<n$ derive to zero. Thus for the Smolin state only $E_{12 \ldots n}$ can be nonzero. To show for which parameters the state is entangled, we need another measure which we introduce in the next section and apply in Sect. VD to the Smolin state.

\section{B. Physical measure}

As we have mentioned, there are many different aspects of multipartite entanglement one might be interested in. The separability measure quantifies the total information content in entanglement and yields an answer to the $\gamma_{k}$-separability in a multipartite state. From a physical point of view, however, we can also present another approach to quantify multipartite entanglement. The basic motivation is to reveal structures of quantum states that go beyond separability. Take for instance the instructive example of a $W$ state $|W\rangle$ and the Greenberger-Horne-Zeilinger state $|G H Z\rangle$ in the three qubit system, where $|W\rangle=1 / \sqrt{3}(|001\rangle+|010\rangle+|100\rangle)$ and $|G H Z\rangle$ as defined in Eq. (24). Both are completely inseparable and thus it is not possible to distinguish them by the separability measure. From a physical point of view the most obvious difference between these two states is the following: Ignoring an arbitrary subsystem will, in case of the $G H Z$ state, yield a mixed separable state, whereas in case of the $W$ state, will yield a mixed entangled state. Any set of entanglement measures that is designed to capture this difference will need a modification in requirements (S2) and (S3) and for mixed density matrices we need the additional requirements (P4) and (P5) as defined below. Thus the properties we propose are the following:

S1a: $\mathcal{E}_{\text {tot }}(\rho)=\sum_{s=1}^{n} S\left(\rho_{s}\right)>0 \quad \forall \rho \quad$ with $k<n$

S1b: $\mathcal{E}_{\text {tot }}(\rho)=0 \quad \forall \rho \quad$ with $k=n$

P2: $\mathcal{E}_{\left\{\alpha_{j}\right\}}(\rho) \geq 0 \quad \forall \quad\left\{\alpha_{j}\right\} \subseteq\left\{\beta_{i}\right\} \in \gamma_{k} \quad$ and $\quad\left|\left\{\alpha_{j}\right\}\right| \geq 2$

P3: $\mathcal{E}_{\left\{\alpha_{j}\right\}}(\rho)=0 \quad \forall \quad\left\{\alpha_{j}\right\} \supset\left\{\beta_{i}\right\} \in \gamma_{k} \quad$ or $\quad\left|\left\{\alpha_{j}\right\}\right|=1$

P4: $\mathcal{E}_{\left\{\alpha_{j}\right\}}\left(\lambda \rho_{1}+(1-\lambda) \rho_{2}\right) \leq \lambda \mathcal{E}_{\left\{\alpha_{j}\right\}}\left(\rho_{1}\right)+(1-\lambda) \mathcal{E}_{\left\{\alpha_{j}\right\}}\left(\rho_{2}\right) \quad$ (convexity)

P5: $\sum_{i} \operatorname{Tr}\left(V_{i} \rho V_{i}^{\dagger}\right) \mathcal{E}_{\text {tot }}\left(\frac{V_{i} \rho V_{i}^{\dagger}}{\operatorname{Tr}\left(V_{i} \rho V_{i}^{\dagger}\right)}\right) \leq \mathcal{E}_{\text {tot }}(\rho)$ (non-increasing on average under LOCC), where $V_{i}$ is a separable operator, i.e. of the local form $V_{i}:=V_{i}^{1} \otimes V_{i}^{2} \otimes \ldots \otimes V_{i}^{n}$. 
Of course capturing this essential difference needs computation of entanglement of all possible subsystems, which are in general mixed. Therefore we propose a set of measures which contain a convex roof extension already for the subsystems of pure multipartite states.

For that let us first define the following useful quantity for any density matrix $\rho=$ $\sum_{i} p_{i}\left|\psi_{i}\right\rangle\left\langle\psi_{i}\right|:$

$$
P(\rho):=\inf _{p_{i}, \psi_{i}, \gamma_{k}} \sum_{i} p_{i}\left(\sum_{s} S\left(\operatorname{Tr}_{\neg s}\left|\psi_{i}\right\rangle\left\langle\psi_{i}\right|\right)\right)
$$

Here the sum over all subsystems $s$ is taken over the entropy of pure states $\psi_{i}$, thus is the "correct" entanglement content of this certain state $\psi_{i}$. Note that we take the infimum also over $\gamma_{k}$. All examples we have considered show that the infimum over $\gamma_{k}$ has not separably to be claimed, because the infimum over all decompositions was always achieved in the correct $\gamma_{k}$-separable decomposition. Moreover, for physical reasons it would be surprising if this was not the case. It would mean that there exist e.g. a partially separable state for which the infimum of the subsystem's entropies was realized for a completely inseparable decomposition. However, we were yet not able to prove that rigorously and therefore we have to conjecture that for any state with the following $\gamma_{k}$ separability

$$
\gamma_{k}:=\left\{\left\{\beta_{1}\right\}\left|\left\{\beta_{2}\right\}\right| \cdots \mid\left\{\beta_{k}\right\}\right\}
$$

the equality

$$
P\left(\rho_{\gamma_{k}}\right)=P\left(\rho_{\left\{\beta_{1}\right\}}\right)+P\left(\rho_{\left\{\beta_{2}\right\}}\right)+(\cdots)+P\left(\rho_{\left\{\beta_{k}\right\}}\right) .
$$

holds. Note that in this way we trivially obtain the additivity property we proposed for the physical measure (see Appendix).

For convenience, we start to define the set of measures for four particles by

$$
\begin{array}{cl}
\text { two-particle entanglement: } & \mathcal{E}_{12}=P\left(\rho_{12}\right), \quad \mathcal{E}_{13}=P\left(\rho_{13}\right) \\
& \mathcal{E}_{14}=P\left(\rho_{14}\right), \quad \mathcal{E}_{23}=P\left(\rho_{23}\right) \\
& \mathcal{E}_{24}=P\left(\rho_{23}\right), \quad \mathcal{E}_{34}=P\left(\rho_{34}\right) \\
\text { three-particle entanglement: } & \mathcal{E}_{123}=\max \left[0, P\left(\rho_{123}\right)-\mathcal{E}_{12}-\mathcal{E}_{13}-\mathcal{E}_{23}\right] \\
& \mathcal{E}_{124}=\max \left[0, P\left(\rho_{124}\right)-\mathcal{E}_{12}-\mathcal{E}_{14}-\mathcal{E}_{24}\right] \\
& \mathcal{E}_{134}=\max \left[0, P\left(\rho_{134}\right)-\mathcal{E}_{13}-\mathcal{E}_{14}-\mathcal{E}_{34}\right] \\
& \mathcal{E}_{234}=\max \left[0, P\left(\rho_{234}\right)-\mathcal{E}_{23}-\mathcal{E}_{24}-\mathcal{E}_{34}\right] \\
\text { four-particle entanglement: } & \mathcal{E}_{1234}=\max \left[0, P\left(\rho_{1234}\right)-\mathcal{E}_{123}-\mathcal{E}_{124}-\mathcal{E}_{134}-\mathcal{E}_{234}\right. \\
& \left.-\mathcal{E}_{12}-\mathcal{E}_{13}-\mathcal{E}_{14}-\mathcal{E}_{23}-\mathcal{E}_{24}-\mathcal{E}_{34}\right]
\end{array}
$$




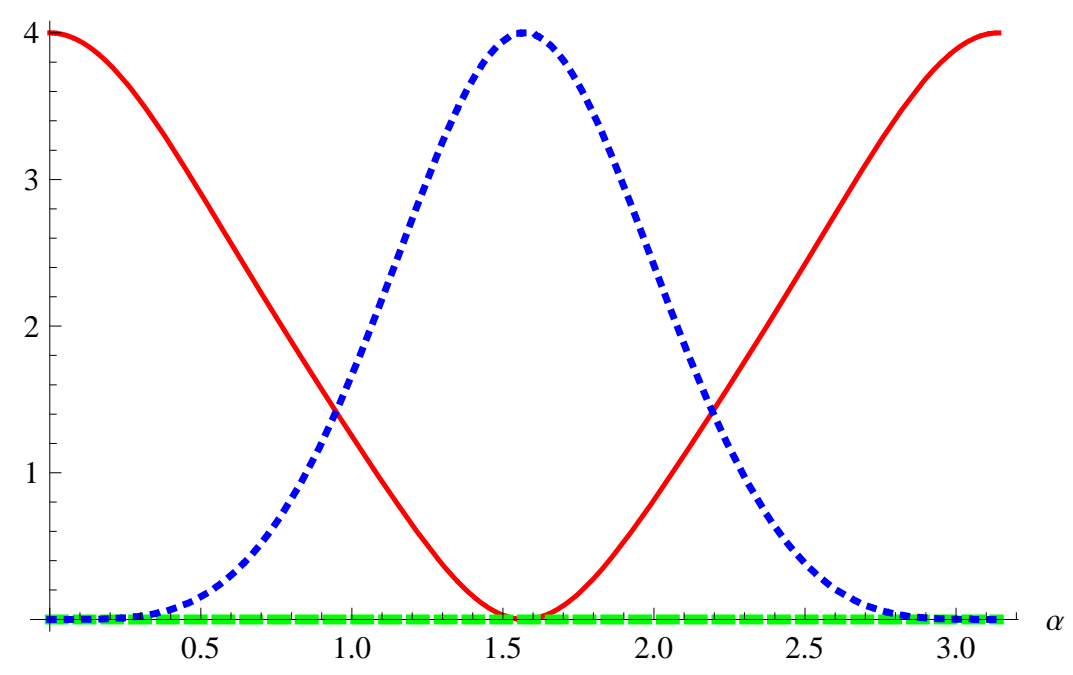

FIG. 2: (Color online) The graphes show the set of the physical measure of the mixture of the $G H Z$ state and the EPR $\otimes E P R$ state, Eq. (40). The solid (red) curve shows the four-partite entanglement $\mathcal{E}_{4}=\mathcal{E}_{1234}$, the dashed (green) curve shows the three-partite entanglement $\mathcal{E}_{3}=$ $\mathcal{E}_{123}+\mathcal{E}_{124}+\mathcal{E}_{134}+\mathcal{E}_{234}$ and the dotted (blue) curve shows the two-partite entanglement $\mathcal{E}_{2}=$ $\mathcal{E}_{12}+\mathcal{E}_{13}+\mathcal{E}_{14}+\mathcal{E}_{23}+\mathcal{E}_{24}+\mathcal{E}_{34}$ in dependence of $\alpha$. The amount of the total entanglement is for the $G H Z$ state and the $E P R \otimes E P R$ state 4, however, in the first case it due to four-partite entanglement whereas in the other case the bipartite entanglement maximizes. The separability measure reveals that the state is $\gamma_{1}=\{1234\}$-separable $\left(E_{1234}=4\right.$, all others zero) except for $\alpha=\frac{\pi}{2}$ then the state is $\gamma_{2}=\{12 \mid 34\}$-separable $\left(E_{t o t}=4, E_{12}=E_{34}=2\right.$ all others zero $)$.

Instructive example for 4 qubits: Consider the mixture of the $G H Z$ state and a pair of EPR-states state:

$$
\rho=\cos ^{2}(\alpha)|G H Z\rangle\left\langle G H Z\left|+\sin ^{2}(\alpha)\right| \Phi^{+}\right\rangle\left\langle\Phi^{+}|\otimes| \Phi^{+}\right\rangle\left\langle\Phi^{+}\right|
$$

with

$$
\begin{aligned}
|G H Z\rangle & =\frac{1}{\sqrt{2}}(|0000\rangle+|1111\rangle) \\
\left|\Phi^{+}\right\rangle & =\frac{1}{\sqrt{2}}(|00\rangle+|11\rangle) .
\end{aligned}
$$

The set of the physical measure is visualized in Fig. 2,

The generalization for any multipartite qudit system is straight forward:

$$
\mathcal{E}_{\left\{\alpha_{j}\right\}}=\max \left[0, P\left(\rho_{\left\{\alpha_{j}\right\}}\right)-\sum_{\left\{\beta_{j}\right\} \subset\left\{\alpha_{j}\right\}} \mathcal{E}_{\left\{\beta_{j}\right\}}\right] .
$$

Note that in case of the physical measure $\sum_{\left\{\alpha_{j}\right\}} \mathcal{E}_{\left\{\alpha_{j}\right\}}=\mathcal{E}_{\text {tot }}(\rho)$ is no longer a requirement. Indeed there exist states which even violate this condition. This is due to the fact that the physical measure quantifies the entanglement of subsystems of a larger systems with respect to possible applications or distillation. In case of overlapping indices of subsystems, e.g. $\rho_{123}$ and $\rho_{124}$ the possibility arises that both share the same entanglement, e.g. in subsystem 1 
and 2, and thus in sum overquantify the actual total entanglement. However, there is no contradiction to possible experiments as one would have to decide, which subsystems to use, e.g. $\rho_{123}$ or $\rho_{124}$, as their entanglement properties cannot be exploited simultaneously.

With the help of the $m$-concurrence, Eq. (12), bounds for every $P(\rho)$ can be computed, see Eq. (18), and thus for the whole set of entanglement measures. In the next section we give further examples and explicit formulae.

\section{FURTHER INSTRUCTIVE EXAMPLES}

In this section we apply our two sets of entanglement measures to certain classes of states and show explicitly how to derive the desired quantities.

\section{A. Two-qubit states}

In the case of pure bipartite qubit states obviously both measures coincide:

$$
\begin{aligned}
E\left(\rho_{12}\right)=E_{12}=\mathcal{E}_{12} & =S\left(\rho_{1}\right)+S\left(\rho_{2}\right) \\
& =-\log _{2}\left(\operatorname{Tr}\left(\rho_{1}^{2}\right)\right)-\log _{2}\left(\operatorname{Tr}\left(\rho_{2}^{2}\right)\right) \\
& =-\log _{2}\left(1-\frac{1}{2} \mathbf{C}_{12}^{2}\right)-\log _{2}\left(1-\frac{1}{2} \mathbf{C}_{12}^{2}\right) \\
& =-2 \log _{2}\left(1-\frac{1}{2} \mathbf{C}_{12}^{2}\right)
\end{aligned}
$$

where the concurrence $\mathbf{C}_{12}$ is twice the Hill-Wootters concurrence [41]. hgbz76 For mixed states $\rho_{12}=\sum_{i} p_{i}\left|\psi_{i}\right\rangle\left\langle\psi_{i}\right|$, we obtain the physical measure by

$$
\begin{aligned}
\mathcal{E}_{12}\left(\rho_{12}\right) & =P\left(\rho_{12}\right)=\inf _{p_{i}, \psi_{i}} \sum p_{i}\left\{S\left(\operatorname{Tr}_{2}\left(\rho_{i}\right)\right)+S\left(\operatorname{Tr}_{1}\left(\rho_{i}\right)\right)\right\} \\
& =2 \inf _{p_{i}, \psi_{i}} \sum p_{i} S\left(\operatorname{Tr}_{2}\left(\rho_{i}\right)\right)=-2 \inf _{p_{i}, \psi_{i}} \sum p_{i} \log _{2}\left(\operatorname{Tr}\left\{\left(\operatorname{Tr}_{2}\left(\rho_{i}\right)\right)^{2}\right\}\right) \\
& =-2 \inf _{p_{i}, \psi_{i}} \sum p_{i} \log _{2}\left(1-\frac{1}{2} \mathbf{C}_{12}^{2}\left(\psi_{i}\right)\right) \\
& \geq-2 \inf _{p_{i}, \psi_{i}} \log _{2}\left(1-\frac{1}{2} \sum p_{i} \mathbf{C}_{12}^{2}\left(\psi_{i}\right)\right)=-2 \log _{2}\left(1-\frac{1}{2} \mathbf{C}_{12}^{2}\left(\rho_{12}\right)\right),
\end{aligned}
$$

where the concurrence can be derived operationally via

$$
\mathbf{C}_{12}\left(\rho_{12}\right)=\max \left\{0,2 \max _{\lambda_{i}^{O_{C}}}\left(\left\{\lambda_{i}^{O_{C}}\right\}\right)-\sum_{i} \lambda_{i}^{O_{C}}\right\}
$$

where the $\lambda_{i}^{O_{C}}$ are the square roots of the eigenvalues of $\rho_{12} \quad \tilde{\rho}_{12}$ and $\tilde{\rho}_{12}=\left(O_{C}+\right.$ $\left.O_{C}^{\dagger}\right) \rho_{12}^{*}\left(O_{C}+O_{C}^{\dagger}\right)$ with $O_{C}+O_{C}^{\dagger}=\sigma_{y} \otimes \sigma_{y}$. For bipartite qubits it is known that there always exists a decomposition such that all concurrences of the pure states $\left|\psi_{i}\right\rangle$ are equal [41], therefore the inequality is in fact an equality and the bounds are also known to be exact. 


\section{B. Two-qutrit states}

In the case of qutrits the linear entropies can be written by only six different operators which are all possible tensor products of the three symmetric Gell-Mann matrices $\sigma^{(i)}=$ $|j\rangle\langle k|+| k\rangle\langle j|$ with $0 \leq j<k \leq 2$ :

$$
\begin{aligned}
& S\left(\rho_{1}\right)=-\log _{2}\left(1-\frac{1}{2}\left(\sum_{i j} \mathbf{C}_{\mathbf{1 2}}^{\sigma^{(i)} \otimes \sigma^{(j)}}\right)\right) \\
& S\left(\rho_{2}\right)=-\log _{2}\left(1-\frac{1}{2}\left(\sum_{i j} \mathbf{C}_{12}^{\sigma^{(i)} \otimes \sigma^{(j)}}\right)\right)
\end{aligned}
$$

Lower bounds on $\mathcal{E}_{12}\left(\rho_{12}\right)=P\left(\rho_{12}\right)$ are then obtained by calculating the squared eigenvalues of all operators $O^{\sigma^{(i)} \otimes \sigma^{(j)}}$ and adding them.

Consider the class of qutrit states which are composed of any two generalized Bell states denoted by $P_{00}, P_{01}$ and the totally mixed state (for an review on the geometry of that class of states see [45, 46, 47])

$$
\rho(\alpha, \beta)=\frac{1-\alpha-\beta}{9} \mathbb{1}+\alpha P_{00}+\beta P_{01} .
$$

Here $P_{k, l}=\left|\Omega_{k, l}\right\rangle\left\langle\Omega_{k, l}\right|$ are obtained by choosing one maximally entangled state, e.g. $\Omega_{00}=$ $\frac{1}{\sqrt{d}} \sum_{s=0}^{d-1}|s\rangle \otimes|s\rangle$, and applying Wely-Operators $W_{k, l}|s\rangle=w^{k(s-l)}|s-l\rangle$ onto one subsystem, i.e. $\Omega_{k, l}=W_{k, l} \otimes \mathbb{1}_{d} \Omega_{0,0}$.

The result of the physical measure is visualized in Fig. 3 (a). If $\alpha$ or $\beta$ is zero, then we obtain the famous isotropic states, for which we now that concurrence increases linearly with $\alpha(\beta)$. If $\alpha$ and $\beta$ are both positive, we observe that not all states negative under partial transpose $(N P T)$ are detected, thus the bounds are not exact. If either $\alpha$ or $\beta$ is zero, the derived bounds detect all NPT states, however, as shown in [45] in this case bound entangled states exist, therefore the bounds are not exact. Recently, by using bounds obtained by an operator acting globally on two copies of a state, these bound entangled states could be detected [48], however, in the region $\alpha, \beta>0$ the bounds did not detect all NPT states either, but they were tighter than the bounds introduced in this work.

Let us consider the class of states

$$
\rho(\alpha, \beta)=\frac{1-\alpha-\beta}{9} \mathbb{1}+\alpha P_{00}+\frac{\beta}{2}\left(P_{01}+P_{02}\right),
$$

where the third Bell states is obtained by applying the same Weyl operator, which transforms $P_{00}$ to $P_{01}$, to $P_{01}$. This class of states are visualized in Fig. 3 (b). Here more symmetries are involved, therefore no bound entangled states can be found and the optimal entanglement witnesses, $\mathcal{K}_{1}, \mathcal{K}_{2}$, correspond to lines. It turns out that the bounds are only exact for $\alpha>\frac{1}{4}$. The requirements for the bounds to be tight is left for further investigation.

\section{Three-qubit states}

Let us consider the most general tripartite pure qubit state,

$$
|\psi\rangle=a|000\rangle+b|001\rangle+c|010\rangle+d|011\rangle+e|100\rangle+f|101\rangle+g|110\rangle+h|111\rangle \text {. }
$$




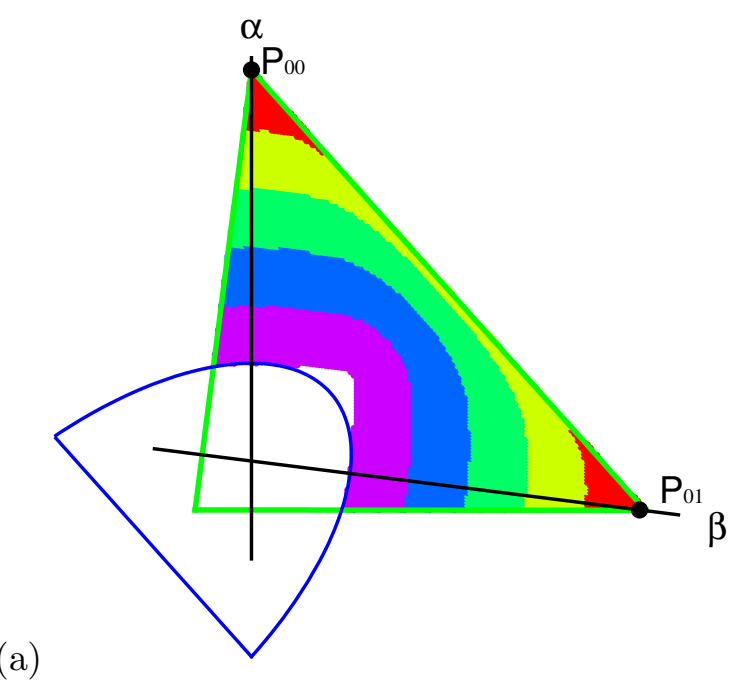

(b)

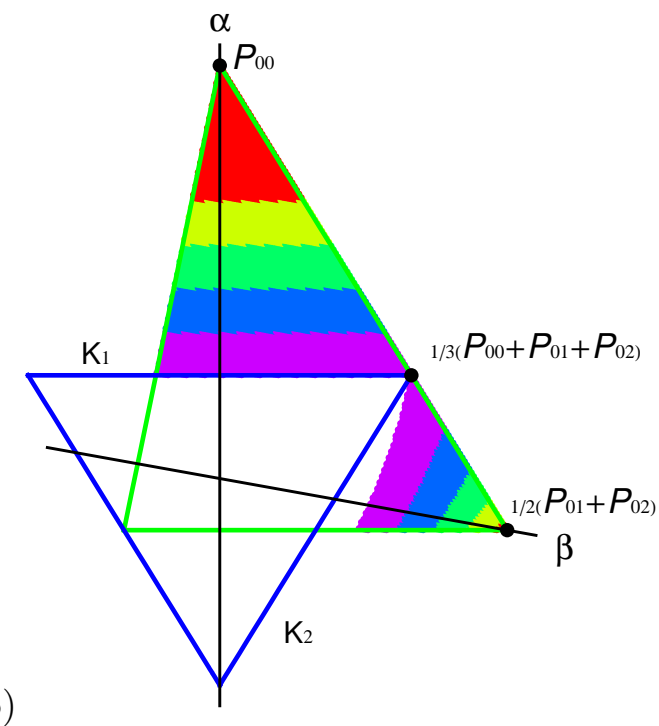

FIG. 3: (Color online) Here two slices through the class of "line" states [47], Eq. (48) and Eq. (49), are shown. The green triangle visualizes the parameter space for which the states are positive, the blue triangle/ellipse the parameter space for which the states are positive under partial transpose (PPT). The colored areas denote the regions where the bounds on the physical measure are nonzero (red: $1 \geq \mathbf{C}>0.8$; yellow: $0.8 \geq \mathbf{C}>0.6$; green: $0.6 \geq \mathbf{C}>0.4$; blue: $0.4 \geq \mathbf{C}>0.2$; purple: $0.2 \geq \mathbf{C}>0$ ). Note that not all states negative under partial transpose are detected. In Fig. (a) for $\alpha<0$ or $\beta<0$ the bound is equivalent to the boundary by PPT, however, as was shown in Ref. [47] a small region of bound entangled states exist in this case. Only for the class of states visualized in Fig. (b) for $\alpha \geq \frac{1}{4}$ the bounds are tight.

The linear entropies of all three subsystems can be rewritten in terms of $m$-concurrences

$$
\begin{aligned}
& S\left(\rho_{1}\right)=-\log _{2}\left(1-\frac{1}{2}\left(\mathbf{C}_{12}^{2}+\mathbf{C}_{13}^{2}+\mathbf{C}_{123}^{2}\right)\right), \\
& S\left(\rho_{2}\right)=-\log _{2}\left(1-\frac{1}{2}\left(\mathbf{C}_{12}^{2}+\mathbf{C}_{23}^{2}+\mathbf{C}_{123}^{2}\right)\right), \\
& S\left(\rho_{3}\right)=-\log _{2}\left(1-\frac{1}{2}\left(\mathbf{C}_{23}^{2}+\mathbf{C}_{13}^{2}+\mathbf{C}_{123}^{2}\right)\right) .
\end{aligned}
$$

Also the entropies of the partially reduced subsystems can be rewritten into the $m^{-}$ concurrences

$$
\begin{aligned}
& S\left(\rho_{12}\right)=-\log _{2}\left(1-\frac{1}{2}\left(\mathbf{C}_{23}^{2}+\mathbf{C}_{13}^{2}+\mathbf{C}_{123}^{2}\right)\right), \\
& S\left(\rho_{13}\right)=-\log _{2}\left(1-\frac{1}{2}\left(\mathbf{C}_{12}^{2}+\mathbf{C}_{23}^{2}+\mathbf{C}_{123}^{2}\right)\right), \\
& S\left(\rho_{23}\right)=-\log _{2}\left(1-\frac{1}{2}\left(\mathbf{C}_{12}^{2}+\mathbf{C}_{13}^{2}+\mathbf{C}_{123}^{2}\right)\right) .
\end{aligned}
$$

Note that for pure tripartite qubit systems there is an intuitive relation between the entropies of the subsystems, $S\left(\operatorname{Tr}_{k}|\psi\rangle\langle\psi|\right)=S\left(\mathbf{T r}_{\neg k}|\psi\rangle\langle\psi|\right)$. For explicit examples see Ref. [40]. 


\section{The generalized Smolin states}

As shown in Ref. [31] for the generalized Smolin state $\rho_{\text {Smolin }}=\frac{1}{2^{n}}\left(\mathbb{1}+\sum c_{i} \sigma_{i}^{\otimes n}\right)(n$ even) only the $n$-flip concurrence $\mathbf{C}_{12 \ldots n}$ is nonzero and the bounds turn out to be tight. In detail one obtains the $n$-partite entanglement $(X=1$ for $n \geq 4$ and $X=2$ for $n=2$ )

$$
\begin{aligned}
E_{12 \ldots n}=\mathcal{E}_{12 \ldots n}= & -4 \log _{2}\left\{\frac { 1 } { 4 } X \operatorname { m a x } \left[0, \frac{1}{2} \max \left\{-1+\vec{c} \cdot \vec{n}^{(1)},-1+\vec{c} \cdot \vec{n}^{(2)},-1+\vec{c} \cdot \vec{n}^{(3)},\right.\right.\right. \\
& \left.\left.\left.-1+\vec{c} \cdot \vec{n}^{(4)}\right\}\right]^{2}\right\}
\end{aligned}
$$

with

$$
\left\{\vec{n}^{(0)}, \vec{n}^{(1)}, \vec{n}^{(2)}, \vec{n}^{(3)}\right\}=\left\{\left(\begin{array}{c}
-1 \\
-1 \\
-1
\end{array}\right),\left(\begin{array}{c}
+1 \\
+1 \\
-1
\end{array}\right),\left(\begin{array}{c}
-1 \\
+1 \\
+1
\end{array}\right),\left(\begin{array}{c}
+1 \\
-1 \\
+1
\end{array}\right)\right\}
$$

Therefore, the state is fully or $n$-separable for $-1+\vec{c} \cdot \vec{n}^{(i)} \leq 0$ for all $i$ and $n$-partite entangled else.

\section{CONCLUSION}

In this paper we first extend the notion of $k$-separability to the $\gamma_{k}$-separability which includes the knowledge which subsystems are inseparable joint. We have pointed out that for mixed states the extension is not straightforward as an ambiguity could arise, however, we could overcome this problem by an appropriate definition, i.e. by a maximization over $k$. Moreover, this novel $\gamma_{k}$-separability concept shows also the desired convexity property for mixtures of different $\gamma_{k}$ states, as visualized in Fig. 1.

Based on this extended concept we could define two different sets of entanglement measures, the first one reveals the $\gamma_{k}$ separability property, the second one reveals the structural, physical properties, e.g. the kind of entanglement.

Both measures are based on the convex roof extension which in general cannot be computed. We use the method of Ref. [40] based on the observation that any entropy can be rewritten by $m$-flip concurrences, i.e. in an operator form. This includes any qudit system. Therefore bounds on the set of measures can be obtained and we show their usefulness in several examples.

For certain applications, such as quantum cryptography scenarios, one is rather interested in the structure of entanglement. For that we have defined a set of measures revealing the two-partite (bipartite), three-partite (tripartite), ..,n-partite entanglement, which we denote as a "physical" measure. It captures for example the different entanglement features of e.g. the $G H Z$ states, the $W$ states or of the $E P R \otimes E P R$ states.

In the last section we gave more instructive examples with explicit formulae to compute lower bounds of the entanglement measures. We show cases where the bounds are surprisingly tight and cases were they are not. Further investigations have to be performed in order to understand in which cases the bounds are equivalent to the infimum of the convex roof.

In summary, we have pointed out that all entanglement features in multipartite systems cannot be revealed by a single set of measures. We defined two sets of measures for multipartite qudit systems and demonstrated its usefulness and computability. Herewith we 
believe one may find novel application exploiting the entanglement of multipartite systems, which is — as this work shows - at least mathematically considerably different to bipartite qubit entanglement.

\section{ACKNOWLEDGEMENTS}

We would like to thank Heide Narnhofer for enlightening discussions on the topic. P. K. would like to acknowledge financial support by FWF project CoQuS No. W1210-N16 of the Austrian Science Foundation.

\section{APPENDIX}

In this appendix we give the proofs that the proposed set of separability measures and the set of the physical measures fulfill the proposed requirements.

\section{A. Proofs for the separability measure}

Here we prove the proposed requirements $\mathrm{S} 2-\mathrm{S} 6$ for the separability measure, S1 is a definition.

\section{Property:}

$$
\text { S2: } \quad E_{\left\{\alpha_{j}\right\}}(\rho)>0 \quad \forall \quad\left\{\alpha_{j}\right\} \in \gamma_{k} \text { and }\left|\left\{\alpha_{j}\right\}\right| \geq 2
$$

with $E_{\left\{\alpha_{j}\right\}}:=\sum_{s \in\left\{\alpha_{j}\right\}}\left(S\left(\rho_{s}\right)-\sum_{\left\{\beta_{j}\right\} \subset\left\{\alpha_{j}\right\}} E_{\left\{\beta_{j}\right\}}\right) \cdot \delta\left[S\left(\rho_{\left\{\alpha_{j}\right\}}\right), 0\right]$.

Proof. Consider a $n$-partite pure state $\rho$ with the separability property $\gamma_{k}:=$ $\left\{\left\{\varepsilon_{1}\right\}\left|\left\{\varepsilon_{2}\right\}\right| \cdots \mid\left\{\varepsilon_{k}\right\}\right\}$. Now if $\left\{\alpha_{j}\right\} \in \gamma_{k}$ then it follows that $\delta\left[S\left(\rho_{\left\{\alpha_{j}\right\}}\right), 0\right]=1$ and consequently $\forall\left\{\beta_{j}\right\} \subset\left\{\alpha_{j}\right\}$ and $\forall\left\{\beta_{j}\right\} \supset\left\{\alpha_{j}\right\}$ is $\delta\left[S\left(\rho_{\left\{\beta_{j}\right\}}\right), 0\right]=0$. Therefore $E_{\left\{\alpha_{j}\right\}}=\sum_{s \in \alpha_{j}} S\left(\rho_{s}\right)>0$ as required.

Property:

$$
S 3: \quad E_{\left\{\alpha_{j}\right\}}(\rho)=0 \quad \forall \quad\left\{\alpha_{j}\right\} \notin \gamma_{k} \quad \text { or } \quad\left|\left\{\alpha_{j}\right\}\right|=1
$$

Proof. Again consider a $n$-partite pure state $\rho$ with the separability property $\gamma_{k}:=$ $\left\{\left\{\varepsilon_{1}\right\}\left|\left\{\varepsilon_{2}\right\}\right| \cdots \mid\left\{\varepsilon_{k}\right\}\right\}$. Now if $\left\{\alpha_{j}\right\} \notin \gamma_{k}$ then it follows that $\delta\left[S\left(\rho_{\left\{\alpha_{j}\right\}}\right), 0\right]=0$ and therefore $E_{\left\{\alpha_{j}\right\}}=0$. If $\left\{\alpha_{j}\right\} \in \gamma_{k}$ but $\left|\left\{\alpha_{j}\right\}\right|=1$ then $E_{\left\{\alpha_{j}\right\}}=S\left(\rho_{\alpha_{j}}\right) \delta\left[S\left(\rho_{\alpha_{j}}\right), 0\right]=0 \cdot 1=0$.

Property:

$$
S 4: E_{\left\{\alpha_{j}\right\}}\left(\rho^{\otimes n}\right)=n E_{\left\{\alpha_{j}\right\}}(\rho)
$$

Proof. Again consider a $n$-partite pure state $\rho$ with the following separability property $\gamma_{k}:=$ $\left\{\left\{\varepsilon_{1}\right\}\left|\left\{\varepsilon_{2}\right\}\right| \cdots \mid\left\{\varepsilon_{k}\right\}\right\} \cdot \rho^{\otimes n}$ must have the same $\gamma_{k}$ property. Thus any nonzero $E_{\left\{\alpha_{j}\right\}}$ will be of the form $E_{\left\{\alpha_{j}\right\}}=\sum_{s=\alpha_{1}}^{\alpha_{j}} S\left(\rho_{s}^{\otimes n}\right)$. Hence it sufficient to prove that

$$
S\left(\rho_{s}^{\otimes n}\right)=n S\left(\rho_{s}\right)
$$

where $S\left(\rho_{s}\right):=\log _{2}\left(\operatorname{Tr}\left(\rho_{s}^{2}\right)\right)$. This is the case as $\operatorname{Tr}\left(\left(\rho_{s}^{\otimes n}\right)^{2}\right)=\left(\operatorname{Tr}\left(\left(\rho_{s}\right)^{2}\right)\right)^{n}$. 


\section{Property:}

$$
S 5: E_{\left\{\alpha_{j}\right\}}\left(U_{\alpha_{1}} \otimes U_{\alpha_{2}} \otimes \cdots \otimes U_{\alpha_{j}} \rho\left(U_{\alpha_{1}} \otimes U_{\alpha_{2}} \otimes \cdots \otimes U_{\alpha_{j}}\right)^{\dagger}\right)=E_{\left\{\alpha_{j}\right\}}(\rho) .
$$

Proof. This proof is trivial as every underlying property $S(\rho):=\log _{2}\left(\operatorname{Tr}\left(\rho^{2}\right)\right)$ is clearly invariant under local unitary transformations, i.e.

$$
\left.\operatorname{Tr}(U \rho \underbrace{U^{\dagger} U}_{\mathbb{1}} \rho U^{\dagger})\right)=\operatorname{Tr}(\rho^{2} \underbrace{U^{\dagger} U}_{\mathbb{1}})=\operatorname{Tr}\left(\rho^{2}\right)
$$

\section{Property:}

$$
S 6: E_{t o t}\left(\rho_{1} \otimes \rho_{2}\right)=E_{t o t}\left(\rho_{1}\right)+E_{t o t}\left(\rho_{2}\right) \quad \text { with } \quad \sum_{\left\{\alpha_{j}\right\}} E_{\left\{\alpha_{j}\right\}}=E_{t o t}=\sum_{s=1}^{n} S\left(\rho_{s}\right) .
$$

Proof. Consider now a $n_{1}$-partite pure state $\rho_{1}$ with the separability property $\gamma_{k_{1}}:=$ $\left\{\left\{\varepsilon_{1}\right\}\left|\left\{\varepsilon_{2}\right\}\right| \cdots \mid\left\{\varepsilon_{k_{1}}\right\}\right\}$ and another $n_{2}$-partite pure state $\rho_{2}$ with the separability property $\gamma_{k_{2}}:=\left\{\left\{\kappa_{1}\right\}\left|\left\{\kappa_{2}\right\}\right| \cdots \mid\left\{\kappa_{k_{2}}\right\}\right\}$. The tensor product of those two states have the separability property

$$
\gamma_{k_{3}}:=\left\{\left\{\varepsilon_{1}\right\}\left|\left\{\varepsilon_{2}\right\}\right| \cdots\left|\left\{\varepsilon_{k_{1}}\right\}\right|\left\{\kappa_{1}\right\}\left|\left\{\kappa_{2}\right\}\right| \cdots \mid\left\{\kappa_{k_{2}}\right\}\right\} .
$$

In this notation the counting of the subsystems of the second system starts with $n_{1}+1$. For every $n$-partite pure state the total entanglement is

$$
E_{t o t}(\rho)=\sum_{s=1}^{n} S\left(\rho_{s}\right)
$$

and hence

$$
E_{\text {tot }}\left(\rho_{1} \otimes \rho_{2}\right)=\sum_{s=1}^{n_{1}+n_{2}} S\left(\rho_{s}\right)=\underbrace{\sum_{s=1}^{n_{1}} S\left(\rho_{s}\right)}_{E_{\text {tot }}\left(\rho_{1}\right)}+\underbrace{\sum_{s=n_{1}+1}^{n_{2}} S\left(\rho_{s}\right)}_{E_{\text {tot }}\left(\rho_{2}\right)} .
$$

\section{B. Proofs for the physical measure}

\section{Property:}

$\mathrm{P} 1: \mathcal{E}_{\left\{\alpha_{j}\right\}}(\rho) \geq 0 \quad \forall \quad\left\{\alpha_{j}\right\} \subseteq \gamma_{k} \wedge\left|\left\{\alpha_{j}\right\}\right| \geq 2$

$\mathrm{P} 2: \mathcal{E}_{\left\{\alpha_{j}\right\}}(\rho)=0 \quad \forall \quad\left\{\alpha_{j}\right\} \supset \gamma_{k} \vee\left|\left\{\alpha_{j}\right\}\right|=1$

Proof. Consider first that

$$
P\left(\rho_{\left\{\alpha_{j}\right\}}\right)=\sum_{\left\{\beta_{m}\right\} \in\left\{\alpha_{j}\right\}} P\left(\rho_{\left\{\beta_{m}\right\}}\right)+P\left(\operatorname{Tr}_{\left\{\beta_{m}\right\} \in\left\{\alpha_{j}\right\}} \rho\right)
$$


and as well

$$
\begin{array}{r}
\sum_{m=2}^{j-1} \sum_{\left\{\epsilon_{m}\right\} \subset\left\{\alpha_{j}\right\}} \mathcal{E}\left(\rho_{\left\{\epsilon_{m}\right\}}\right) \geq \sum_{\left\{\beta_{m}\right\} \in\left\{\alpha_{j}\right\}} P\left(\rho_{\left\{\beta_{m}\right\}}\right)+P\left(\operatorname{Tr}_{\left\{\beta_{m}\right\} \in\left\{\alpha_{j}\right\}} \rho\right) \\
\text { iff } \quad \exists\left\{\beta_{m}\right\} \in\left\{\alpha_{j}\right\}
\end{array}
$$

such that the difference derives to

$$
\begin{array}{r}
P\left(\rho_{\left\{\alpha_{j}\right\}}\right)-\sum_{m=2}^{j-1} \sum_{\left\{\epsilon_{m}\right\} \subset\left\{\alpha_{j}\right\}} \mathcal{E}_{\left\{\epsilon_{m}\right\}}(\rho) \leq 0 \\
\text { iff } \exists\left\{\beta_{m}\right\} \in\left\{\alpha_{j}\right\}
\end{array}
$$

from which consequently follows

$$
\mathcal{E}_{\left\{\alpha_{j}\right\}}(\rho)=0 \quad \forall \quad\left\{\alpha_{j}\right\} \supset \gamma_{k} \text { or }\left|\left\{\alpha_{j}\right\}\right|=1
$$

\section{Property:}

P3: $\mathcal{E}_{\left\{\alpha_{j}\right\}}\left(\rho^{\otimes n}\right)=n \mathcal{E}_{\left\{\alpha_{j}\right\}}(\rho)$ (additivity on copies of the same state)

Proof. Additivity follows directly if the conjecture is valid, i.e.

$$
\mathcal{E}_{\left\{\alpha_{j}\right\}}\left(\rho^{\otimes n}\right)=\mathcal{E}_{\left\{\alpha_{j}\right\}}\left(\rho^{\prime}\right)
$$

where the separability property of $\rho^{\prime}$ is

$$
\gamma_{k}^{\prime}=\left\{\left\{\gamma_{k}\right\}\left|\left\{\gamma_{k}\right\}\right|(\cdots) \mid\left\{\gamma_{k}\right\}\right\}
$$

so the infimum is achieved in the appropriate decomposition such that

$$
\mathcal{E}_{\left\{\alpha_{j}\right\}}\left(\rho^{\otimes n}\right)=n \mathcal{E}_{\left\{\alpha_{j}\right\}}(\rho) .
$$

\section{Property:}

$\mathrm{P} 4: \mathcal{E}_{\left\{\alpha_{j}\right\}}\left(\lambda \rho_{1}+(1-\lambda) \rho_{2}\right) \leq \lambda \mathcal{E}_{\left\{\alpha_{j}\right\}}\left(\rho_{1}\right)+(1-\lambda) \mathcal{E}_{\left\{\alpha_{j}\right\}}\left(\rho_{2}\right) \quad$ (convexity)

Proof. Invariance under local unitaries is easy to prove as the constituting functions $P(\rho)$ are themselves invariant under local unitary transformations

$$
P(\rho):=\inf _{p_{i}, \psi_{i}} \sum_{i} p_{i}\left(\sum_{s} S\left(\operatorname{Tr}_{\neg s}\left|\psi_{i}\right\rangle\left\langle\psi_{i}\right|\right)\right)
$$

and therefore

$$
\begin{array}{r}
\operatorname{Tr}_{\neg s}\left(U_{\alpha_{1}} \otimes U_{\alpha_{2}} \otimes \cdots \otimes U_{\alpha_{j}}\left|\psi_{i}\right\rangle\left\langle\psi_{i}\right|\left(U_{\alpha_{1}} \otimes U_{\alpha_{2}} \otimes \cdots \otimes U_{\alpha_{j}}\right)^{\dagger}\right) \\
=\operatorname{Tr}_{\neg s}(\underbrace{\left(U_{\alpha_{1}} \otimes U_{\alpha_{2}} \otimes \cdots \otimes U_{\alpha_{j}}\right)^{\dagger} U_{\alpha_{1}} \otimes U_{\alpha_{2}} \otimes \cdots \otimes U_{\alpha_{j}}}_{\mathbb{1}}\left|\psi_{i}\right\rangle\left\langle\psi_{i}\right|)
\end{array}
$$

such that

$$
P\left(U_{\alpha_{1}} \otimes U_{\alpha_{2}} \otimes \cdots \otimes U_{\alpha_{j}} \rho\left(U_{\alpha_{1}} \otimes U_{\alpha_{2}} \otimes \cdots \otimes U_{\alpha_{j}}\right)^{\dagger}\right)=P(\rho)
$$




\section{Property:}

P5: $\mathcal{E}_{\left\{\alpha_{j}\right\}}\left(\lambda \rho_{1}+(1-\lambda) \rho_{2}\right) \leq \lambda \mathcal{E}_{\left\{\alpha_{j}\right\}}\left(\rho_{1}\right)+(1-\lambda) \mathcal{E}_{\left\{\alpha_{j}\right\}}\left(\rho_{2}\right)$ (Convexity)

Proof. To prove that the total entanglement $E_{\text {tot }}(\rho)$ is convex one needs to prove that $P(\rho)$ is convex, which is trivial, as:

$$
\begin{aligned}
& P\left(\lambda \rho_{1}+(1-\lambda) \rho_{2}\right) \\
= & \inf _{p_{i}, r_{j}, \psi_{i}, \phi_{j}, \gamma_{n}, \tau_{m}}\left(\sum_{i} p_{i} \lambda \sum_{s} S\left(\operatorname{Tr}_{\neg_{s}}\left|\psi_{i}\right\rangle\left\langle\psi_{i}\right|\right)+\sum_{j} r_{j}(1-\lambda) \sum_{s} S\left(\operatorname{Tr}_{\urcorner_{s}}\left|\phi_{j}\right\rangle\left\langle\phi_{j}\right|\right)\right) \\
\leq & \underbrace{\inf _{p_{i}, \psi_{i}, \gamma_{n}}\left(\sum_{i} p_{i} \lambda \sum_{s} S\left(\operatorname{Tr}_{\neg_{s}}\left|\psi_{i}\right\rangle\left\langle\psi_{i}\right|\right)\right)}_{\lambda P\left(\rho_{1}\right)}+\underbrace{\inf _{r_{j}, \phi_{j}, \tau_{m}}\left(\sum_{j} r_{j}(1-\lambda) \sum_{s} S\left(\operatorname{Tr}_{\neg_{s}}\left|\phi_{j}\right\rangle\left\langle\phi_{j}\right|\right)\right)}_{(1-\lambda) P\left(\rho_{2}\right)}
\end{aligned}
$$

Note that the constituting elements $E_{\left\{\alpha_{j}\right\}}(\rho)$ are only convex under local combinations of the form

$$
\begin{aligned}
\rho \underbrace{+}_{\text {local }} \sigma: & =\lambda \sum_{i} p_{i}\left(\rho_{\left\{\beta_{1}\right\}}^{i} \otimes \rho_{\left\{\beta_{2}\right\}}^{i} \otimes(\cdots) \rho_{\left\{\beta_{k}\right\}}^{i}\right)+(1-\lambda) \sum_{i} p_{i}\left(\sigma_{\left\{\beta_{1}\right\}}^{i} \otimes \rho_{\left\{\beta_{2}\right\}}^{i} \otimes(\cdots) \rho_{\left\{\beta_{k}\right\}}^{i}\right) \\
& =\sum_{i} p_{i}\left(\left(\lambda \rho_{\left\{\beta_{1}\right\}}^{i}+(1-\lambda) \sigma_{\left\{\beta_{1}\right\}}^{i}\right) \otimes \rho_{\left\{\beta_{2}\right\}}^{i} \otimes(\cdots) \rho_{\left\{\beta_{k}\right\}}^{i}\right)
\end{aligned}
$$

\section{Property:}

P6: $\sum_{i} \operatorname{Tr}\left(V_{i} \rho V_{i}^{\dagger}\right) \mathcal{E}_{t o t}\left(\frac{V_{i} \rho V_{i}^{\dagger}}{\operatorname{Tr}\left(V_{i} \rho V_{i}^{\dagger}\right)}\right) \leq \mathcal{E}_{\text {tot }}(\rho)$ (non-increasing on average under LOCC),

Proof. This has already been proven, see e.g. Ref. [49].

[1] J. S. Bell, Physics 1, 195 (1964).

[2] J. F. Clauser, M. A. Horne, A. Shimony, and R. A. Holt, Phys. Rev. Lett. 23, 880 (1969).

[3] R. Bertlmann, W. Grimus, and B. Hiesmayr, Phys. Rev. A 372, 3608 (2006).

[4] B. Hiesmayr, Eur. Phys. J. C 50, 73 (2007).

[5] M. Nielsen and I. Chuang, Quantum Computation and Quantum Information (Cambridge University Press, Cambridge, England, 2000).

[6] D. Bouwmeester, A. Ekert, and A. Zeilinger, eds., The physics of quantum information: quantum cryptography, quantum teleportation, quantum computation (Springer, Berlin, Heidelberg, New York, 2000).

[7] R. A. Bertlmann and A. Zeilinger, eds., Quantum [un]speakables, from Bell to quantum information (Springer, Berlin Heidelberg New York, 2002).

[8] D. Bruß, J. Math. Phys. 43, 4237 (2002).

[9] R. Horodecki, P. Horodecki, M. Horodecki, and K. Horodecki, e-print arXiv:quant-ph/0702225.

[10] W. Dür, G. Vidal, and J. I. Cirac, Phys. Rev. A 62, 062314 (2000). 
[11] F. Verstraete, J. Dehaene, B. D. Moor, and H. Verschelde, Phys. Rev. A 65, 052112 (2002).

[12] F. Verstraete, J. Dehaene, and B. D. Moor, Phys. Rev. A 68, 012103 (2003).

[13] V. Coffman, J. Kundu, and W. K. Wootters, Phys. Rev. A 61, 052306 (2000).

[14] C. H. Bennett, S. Popescu, D. Rohrlich, J. A. Smolin, and A. V. Thapliyal, Phys. Rev. A 63, 012307 (2000).

[15] C. Kruszynska and B. Kraus, e-print arXiv:0808.3862.

[16] D. A. Meyer and N. R. Wallach, J. Math. Phys. 43, 4273 (2002).

[17] D. Teresi, A. Napoli, and A. Messina, e-print arXiv:0805.4345.

[18] G. Rigolin, T. R. de Oliveira, and M. C. de Oliveira, Phys. Rev. A 74, 022314 (2006).

[19] R. Somma, G. Ortiz, H. Barnum, E. Knill, and L. Viola, Phys. Rev. A 70, 042311 (2004).

[20] H. Barnum, E. Knill, G. Ortiz, R. Somma, and L. Viola, Phys. Rev. Lett. 92, 107902 (2004).

[21] R. Demkowicz-Dobrzanski, A. Buchleitner, M. Kus, and F. Mintert, Phys. Rev. A 74, 052303 (2006).

[22] J. Kempe, Phys. Rev. A 60, 910 (1999).

[23] V. Scarani and N. Gisin, Phys. Rev. Lett. 87, 117901 (2001).

[24] N. J. Cerf, S. Massar, and S. Schneider, Phys. Rev. A 66, 042309 (2002).

[25] C. Kruszynska, S. Anders, W. Dür, and H. J. Briegel, Phys. Rev. A 73, 062328 (2006).

[26] Y. Shi, quant-ph/0201079.

[27] A. Uhlmann, Phys. Rev. A 62, 032307 (2000).

[28] P. Rungta, V. Buek, C. M. Caves, M. Hillery, and G. J. Milburn, Phys. Rev. A 64, 042315 (2001).

[29] K. Audenaert, F. Verstraete, and B. D. Moor, Phys. Rev. A 64, 052304 (2001).

[30] F. Mintert, A. R. R. Carvalho, M. Kus, and A. Buchleitner, Phys. Rep. 415, 207 (2005).

[31] B. C. Hiesmayr, F. Hipp, M. Huber, P. Krammer, and C. Spengler, Phys. Rev. A 78, 042327 (2008).

[32] R. Augusiak and P. Horodecki, Phys. Rev. A 74, 010305 (2006).

[33] J. Smolin, Phys. Rev. A 63, 032112 (2001).

[34] C. H. Bennett, G. Brassard, S. Popescu, B. Schumacher, J. A. Smolin, and W. K. Wootters, Phys. Rev. A 53, 2046 (1996).

[35] C. H. Bennett, D. P. DiVincenzo, J. A. Smolin, and W. K. Wootters, Phys. Rev. A 54, 3824 (1996).

[36] V. Vedral, M. B. Plenio, M. A. Rippin, and P. L. Knight, Phys. Rev. Lett. 78, 2275 (1997).

[37] V. Vedral and M. B. Plenio, Phys. Rev. A 57, 1619 (1998).

[38] A. Rényi, Proceedings of the 4th Berkeley Symposium on Mathematics, Statistics and Probability, p. 547 (1960).

[39] A. Uhlmann, Phys. Rev. A 62, 032307 (2008).

[40] B. Hiesmayr and M. Huber, Phys. Rev. A 78, 012342 (2008).

[41] S. Hill and W. K. Wootters, Phys. Rev. Lett. 78, 5022 (1997).

[42] W. K. Wootters, Phys. Rev. Lett. 80, 2245 (1998).

[43] G. Kimura, Phys. Lett. A 314, 339 (2003).

[44] R. A. Bertlmann and P. Krammer, J. Phys. A: Math. Theor. 41, 235303 (2008).

[45] B. Baumgartner, B. Hiesmayr, and H. Narnhofer, Phys. Rev. A 74, 032327 (2006).

[46] B. Baumgartner, B. Hiesmayr, and H. Narnhofer, J. Phys. A 40, 7919 (2006).

[47] B. Baumgartner, B. Hiesmayr, and H. Narnhofer, Physics Letters A 372, 2190 (2008).

[48] J. Bae, M. Tiersch, S. Sauer, F. de Melo, F. Mintert, B. Hiesmayr, and A. Buchleitner (2009), quant-ph/0902.4372. 
[49] M. Plenio and S. Virmani, Quant. Inf. Comp. 7, 1 (2007). 